\title{
Clinical Stage IIA Esophageal Adenocarcinoma AJCC v8
}

National Cancer Institute

\section{Source}

National Cancer Institute. Clinical Stage IIA Esophageal Adenocarcinoma A/CC v8. NCI

Thesaurus. Code C133405.

Stage IIA includes: T1, N1, M0. T1: Tumor invades the lamina propria, muscularis mucosae, or submucosa. N1: T umor with metastasis in one or two regional lymph nodes. M0: No distant metastasis. (AJCC 8th ed.) 